\title{
Measuring informed choice in population-based reproductive genetic screening: a systematic review
}

\author{
Alice Grace Ames ${ }^{1,2}$, Sylvia Ann Metcalfe ${ }^{\star, 1,2}$, Alison Dalton Archibald ${ }^{1,2,3}$, Rony Emily Duncan ${ }^{2,4,5}$ \\ and Jon Emery ${ }^{6}$
}

Genetic screening and health-care guidelines recommend that programmes should facilitate informed choice. It is therefore important that accurate measures of informed choice are available to evaluate such programmes. This review synthesises and appraises measures used to evaluate informed choice in population-based genetic screening programmes for reproductive risk. Databases were searched for studies offering genetic screening for the purpose of establishing reproductive risk to an adult population sample, in which aspects of informed choice were measured. Studies were included if, at a minimum, measures of uptake of screening and knowledge were used. Searches identified 1462 citations and 76 studies were reviewed in full text; 34 studies met the inclusion criteria. Over 20 different measures of informed choice were used. Many measures lacked adequate validity and reliability data. This systematic review will inform future evaluation of informed choice in population genetic screening programmes.

European Journal of Human Genetics (2015) 23, 8-21; doi:10.1038/ejhg.2014.89; published online 21 May 2014

\section{INTRODUCTION}

Population-based reproductive genetic screening programmes offer individuals and couples without a specific family history or predisposition the opportunity to learn if they have an increased risk of having a child with a particular genetic condition or conditions in current or future pregnancies. Developments in genetic health care are constantly evolving, with screening technology becoming publically and privately available such as multiplex panels for carrier screening, whole exome/genome sequencing and noninvasive prenatal testing.

Genetic screening guidelines recommend that ethical and psychosocial aspects of screening programmes, such as informed choice, ${ }^{1-3}$ are evaluated in addition to technical aspects such as analytical and clinical validity. ${ }^{3}$ Screening decisions do not always have a clear medical imperative or benefit that outweighs the potential for psychological harms for the individual. ${ }^{4}$ Therefore, screening programmes should not directly encourage uptake of screening or influence reproductive decision making following a screen-positive result. ${ }^{5}$ The decision of whether to accept screening resides with the individual and it is therefore important that the decision is informed, based on correct knowledge and free of coercion from others. ${ }^{1,3,5}$ Thus, the success of population genetic screening programmes should not be based solely on the uptake of screening, and it is important to evaluate whether these programmes are in fact facilitating informed choice. $^{2}$

Evaluation of genetic screening programmes requires accurate, validated measures of informed choice, which is a complex, multidimensional construct, defined in various ways throughout the literature. ${ }^{6-12}$ Informed choice may literally be measured through evaluation of knowledge and uptake of screening, to indicate whether the decision to accept or decline screening was 'informed'. However, definitions of informed choice more commonly involve at least two elements: having a factual understanding and making a choice aligned with one's values. ${ }^{12,13}$ Some definitions distinguish between choice and decision making with the inclusion of deliberation and the behavioural implementation of the decision to reflect the cognitive processes involved in decision making. ${ }^{7}, 10$ However, underlying these various definitions is the same concept of an informed and autonomous choice. ${ }^{13,14}$ The complexity of measuring informed choice, because of the different ways informed choice has been defined and measured in a range of screening settings, has been recognised. ${ }^{12,14-16}$

Here we present the findings of a systematic review focussing on reproductive genetic screening in the general population, where screening was used to assess risk of a genetic condition in current or future pregnancies. A variety of conditions are included such as Down syndrome (DS) and single gene disorders. Screening choices in any of these scenarios can be complex, as individuals' choices depend on the value they place on the benefits and harms of screening. Aspects of the condition also influence the way in which screening is offered, for example, screening a fetus for DS, a genetic but not usually inherited condition, is common and offered during each pregnancy in many countries. On the other hand, population genetic screening for carrier status, which provides an individual with reproductive risk, needs only to be performed once in a person's lifetime and so can be offered anytime, including preconceptionally or during pregnancy.

Although there have been many studies evaluating population reproductive genetic screening, this review focusses on studies that

${ }^{1}$ Genetics Education and Health Research, Murdoch Childrens Research Institute, Parkville, Victoria, Australia; ${ }^{2}$ Department of Paediatrics, The University of Melbourne, Melbourne, Victoria, Australia; ${ }^{3}$ Victorian Clinical Genetics Services, Melbourne, Victoria, Australia; ${ }^{4}$ Centre for Adolescent Health, Murdoch Childrens Research Institute, Parkville, Victoria, Australia; ${ }^{5}$ Health Services Delivery for Adolescents, Murdoch Childrens Research Institute, Parkville, Victoria, Australia; ${ }^{6}$ General Practice and Primary Care Academic Centre, University of Melbourne, Parkville, Victoria, Australia

*Correspondence: Professor SA Metcalfe, Genetics Education and Health Research, Murdoch Childrens Research Institute, Royal Children's Hospital, Flemington Road, Parkville, Victoria 3052, Australia. Tel: +61 38341 6309; Fax: +61 38341 6212; E-mail: sylvia.metcalfe@mcri.edu.au

Received 3 December 2013; revised 11 March 2014; accepted 10 April 2014; published online 21 May 2014 
specifically aimed to measure informed choice. This work will inform future research aiming to evaluate informed choice in genetic screening programmes. This systematic review investigates how informed choice has been measured in population reproductive genetic screening, what variables, methods and validation procedures have been used, and what rates of informed choice have been reported in these studies.

\section{MATERIALS AND METHODS}

Databases indexing medical and psychosocial research were searched for studies from the past 30 years (1982 to September 2012), addressing informed choice in population-based genetic carrier or DS screening. The search strategy was based on: (1) genetic screening, (2) measurement, and (3) informed choice. Search terms were defined using key words and thesaurus terms specific to Medline, Embase, Cochrane Library, CINHAL, and PsycInfo databases that were searched using this strategy (see Supplementary Table 1).

\section{Inclusion criteria}

Studies were included if they met the following criteria: original research during the past 30 years; published in English; adults or pregnant women from an unselected, population-based sample; the study needed to be in the context of offering a genetic test to determine reproductive risk; the study needed to have reported on measures of informed choice obtained prospectively at the time of test offer, including uptake of screening and knowledge as a minimum requirement to evaluate informed choice. Genetic testing is defined here according to the Foundation for Genomics and Population Health as: 'a test to detect the presence or absence of, or change in, a particular gene or chromosome'; the test did not need to have been a DNA or chromosomal assay, for example, it could be a full blood examination to identify thalassaemia carriers or ultrasound screening to identify markers for DS. ${ }^{17}$

Studies were excluded if they: were reviews, opinion or commentary, letters to the editor, guidelines, or used only qualitative methodology; used a hypothetical scenario; sampled selected subgroups of the population, such as those identified in high-risk categories through first-trimester screening for DS and trisomy 18, a group affected by, or with family history of, a genetic condition, or those undergoing preimplantation genetic diagnosis as part of in vitro fertilisation treatment; reported fetal anomaly scans, or other tests for structural anomalies without specifically reporting on risk of DS for the pregnancy. The cited references of the included papers were searched for additional relevant titles. Corresponding authors of the included papers and experts in the field were contacted for recommendations of any additional relevant papers.

\section{RESULTS}

The review of titles and abstracts was conducted by AGA, and JE confirmed the application of the exclusion criteria in 10\% of papers (see Figure 1). During data extraction, eight papers were excluded either because they measured informed choice many months after test offer ${ }^{18,19}$ or did not report uptake of screening. ${ }^{20-25}$ In total, 34 articles met the inclusion criteria; data were extracted for 33 articles as two studies reported the same data on informed choice. ${ }^{26,27}$ The final inclusion of papers was confirmed by the authors JE and SAM.

\section{Summary of studies}

Of the 33 studies included in the review, less than half $(n=11)$ of the studies were randomised control trials (RCTs, Tables 1-4). ${ }^{11,13,26,28-35}$ Twenty-two studies were prospective, ${ }^{12,28,36-55}$ including cross-sectional, ${ }^{41,42,49}$ quasi-experimental, ${ }^{53}$ pilot, ${ }^{50}$ and longitudinal study designs. ${ }^{52}$ Of the RCTs, five involved an information or educational intervention, ${ }^{28,29,32-34}$ five randomised the screening offer, ${ }^{11,13,26,30,35}$ and there was one decision aid trial. ${ }^{31}$ The 33 studies were published between $1991,{ }^{54}$ and 2012, ${ }^{36}$ with 25 studies published between 2002 and 2012. Three studies used the same cohort as in previous studies; ${ }^{13,39,46}$ in subsequent studies the sample was stratified differently, ${ }^{40}$ different measures were applied, ${ }^{11}$ and additional validation data were reported. ${ }^{45}$ Eight studies were conducted in continental Europe, ${ }^{11,13,38,43-46,53}$ and 14 were from the United Kingdom. ${ }^{12,26,29,30,32-35,37,39-41,51,54}$ Seven studies were from Australia, ${ }^{31,36,48-50,52,55}$ three from the United States, ${ }^{28,42,56}$ and one from Taiwan. ${ }^{47}$ Prenatal screening for DS was the most common screening programme evaluated (27 studies). ${ }^{11-13,28,29,31-35,37-40,42-49,51-53,55,56}$ Three studies offering population carrier screening in prenatal settings included screening single gene disorders: sickle cell disease and thalassaemia (haemoglobinopathies $(\mathrm{Hb}))^{26,41}$ and cystic fibrosis $(\mathrm{CF}){ }^{30}$ Of the 33 studies reviewed, only one included partners of the pregnant women, ${ }^{30}$ and one offered CF carrier screening to men as well as women. ${ }^{54}$ Only two studies offered carrier screening to a nonpregnant population, for $\mathrm{CF}^{54}$ and fragile $\mathrm{X}$ syndrome (FXS). ${ }^{50}$

Uptake of screening varied in the DS studies from $13 \%{ }^{44}$ to $91.6 \% .^{38}$ Six DS studies reported $100 \%$ uptake; ${ }^{42,45-49}$ however, these studies only included women who had accepted screening as they were recruited at their screening consultation. High rates of screening uptake were reported for prenatal $\mathrm{Hb}$ screening in two studies $\left(100 \%{ }^{41}\right.$ and $\left.90.7 \%{ }^{26}\right)$. Similar uptake rates were reported in CF carrier screening in a prenatal setting $\left(89-91 \%^{30}\right.$ ) and a preconception setting (89\%, family planning clinic site) ${ }^{54}$ However, the two preconception screening studies $\left(\mathrm{CF}^{54} \text { and FXS }\right)^{50}$ reported that uptake varied with recruitment method and site..$^{50,54}$ Studies reporting $100 \%$ uptake, however, should not be considered representative as they either only included individuals already having screening, ${ }^{47}$ or recruited from a site where women were presenting for prenatal screening. ${ }^{42,45,46,48,49}$

\section{MEASURES OF INFORMED CHOICE}

\section{Knowledge}

As determined by the inclusion criteria, all of the 33 studies measured and reported on knowledge, a core component of informed choice. The knowledge items used in these studies measured knowledge recall, although some studies also measured perceived knowledge ${ }^{47,56}$ and risk accuracy. ${ }^{56}$ Throughout these studies the type and number of knowledge items varied, and knowledge levels were not generally comparable across studies. Two validated DS knowledge scales were used in more than one study; six studies used the scale developed by Marteau et al $12,31,39,40,49,52,55$ and two studies used the scale by Dormandy et al, $34,44,45$ whereas the other DS studies each used their own specific knowledge scale. Studies screening for single gene disorders developed condition-specific knowledge scales for FXS, 50 $\mathrm{Hb}^{26,41}$ and $\mathrm{CF}^{30,54}$ Knowledge results were reported in different ways using means, ${ }^{11,12,29,32,34,36,38,40,41,51-53,55,56}$ medians, ${ }^{26,28,35,36}$ dichotomised cutoffs of good knowledge, ${ }^{11,13,26,31,36,39,43-46,49,55}$ categories, ${ }^{32,37,45,46,48,50}$ and percentage of items answered correctly. ${ }^{28,30,42,47,54}$

\section{OTHER MEASURES OF INFORMED CHOICE}

Measures of informed choice other than knowledge were heterogeneous, involving a variety of different aspects such as perceptions of knowledge and information, ${ }^{32,37,43,49,56}$ the multidimensional measure of informed choice (MMIC), ${ }^{11-13,26,31,35,36,39-41,43-46,49,52}$ perceptions of choice, ${ }^{32,43,49,51,56}$ attitudes and feelings towards screening, ${ }^{30,43,47,50,56}$ decisional conflict, ${ }^{11,28,31,38,56}$ satisfaction and decisional regret, ${ }^{11,13,28,29,32}$ and systematic and informed decision making. ${ }^{29,42,51}$ 


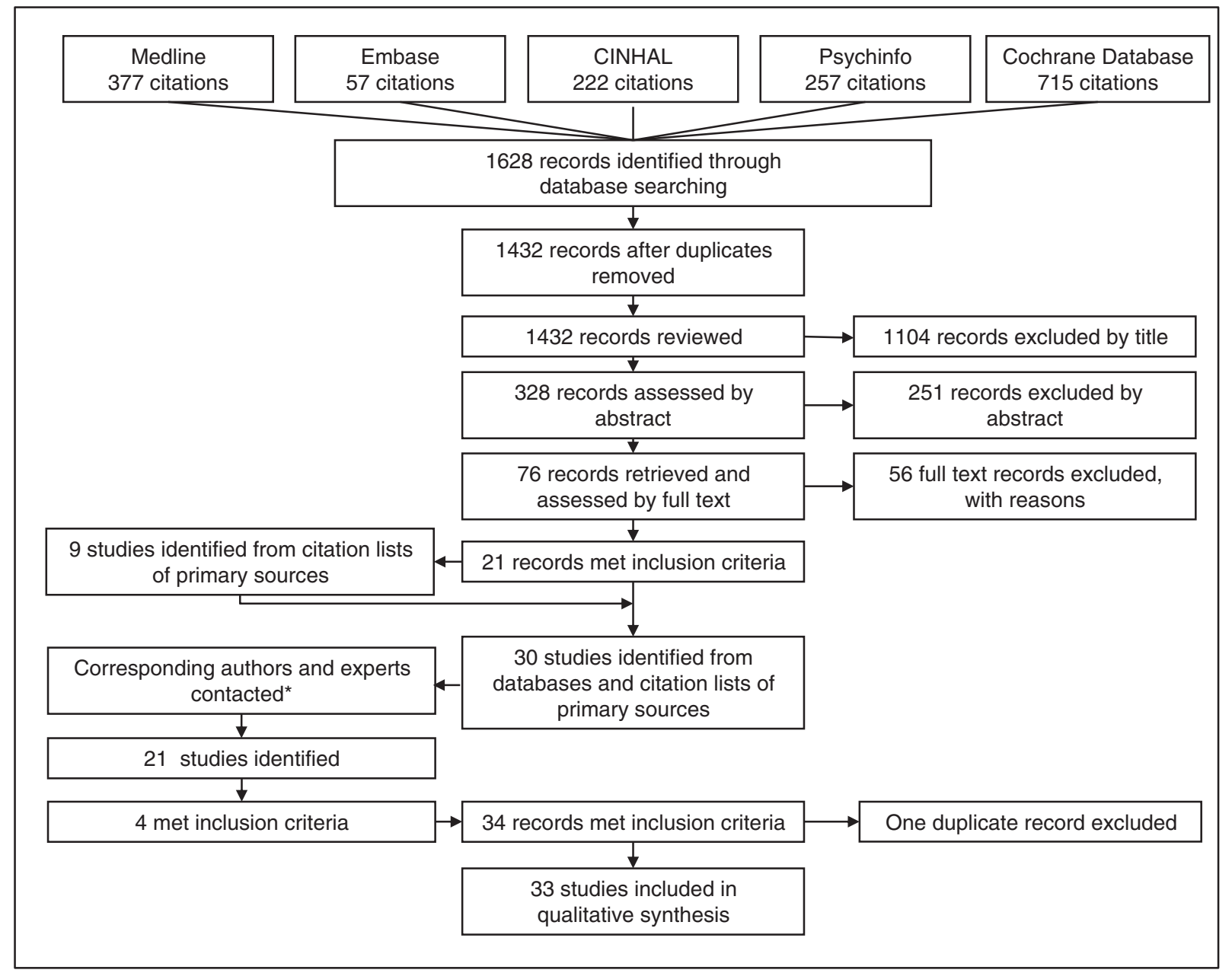

Figure 1 Flow diagram of systematic review search results. *All corresponding authors (30) and 3 experts were contacted. Of the 21 studies identified, 19 were from corresponding authors and 2 from experts. The four studies meeting inclusion criteria were identified by corresponding authors (two) and by experts (two).

\section{Perceptions of knowledge and information}

Two studies measured perception of knowledge, ${ }^{47,56}$ and five studies measured perception of the information provided. ${ }^{32,37,43,49,53}$ Measuring participants' perception of knowledge and information is another way of assessing the quality and level of information provided in a screening study. These measures directly asked how women felt about the information provided, ${ }^{53}$ and if they were provided with enough information, ${ }^{49}$ with the purpose to inform how to present information in a meaningful way to aid participants' understanding and facilitate informed choice. One study used a single item of perceived adequacy of information as their primary outcome measure, and showed that $\sim 60 \%$ of participants in both trial arms reported that there was adequate information to make a choice about screening. ${ }^{32}$ Another study showed informed choice was dependent on a measure of the quality of the information provided $(P<0.001)^{43}$ and that less satisfactory information was related to the perception of the screening being compulsory rather than optional. ${ }^{43}$

\section{Multidimensional measure of informed choice}

The MMIC was the most widely used measure, and was used in 17 studies, ${ }^{11-13,26,31,35,36,39-41,43-46,49,52,55}$ with the majority $(n=14)$ assessing informed choice in prenatal screening for DS in the United Kingdom, continental Europe, and Australia
(Table 1). ${ }^{11-13,31,35,39,40,43-46,49,52,55}$ The MMIC was adapted for use in population carrier screening for $\mathrm{Hb}$ in pregnancy by using a specific knowledge scale for $\mathrm{Hb},{ }^{26}$ and for low literacy populations, ${ }^{41}$ as well as for FXS carrier screening in a preconception setting (Table 3). ${ }^{36,50}$

The MMIC is a theory-based measure, modelling the attitudesbehaviour domain on the Theory of Planned Behaviour. ${ }^{57}$ The MMIC is based on the definition of informed choice being 'one that is based on relevant knowledge, consistent with the decision-maker's values and behaviourally implemented.' ${ }^{2}$ Some of the studies in this review were published before the MMIC, and hence they did not use the same terminology for defining informed choice as the authors of the MMIC. ${ }^{29,30,33,34,51}$

The MMIC combines knowledge with 'value-consistency' comprising attitudes and screening behaviour into a single measure. A choice is value consistent when an individual has positive attitudes and accepts screening or has negative attitudes and declines screening. A value consistent choice becomes an informed choice when made with good knowledge. All other combinations are classified as uninformed choices.

Each continuous knowledge and attitude scale is dichotomised into 'good'/'poor' knowledge and positive/negative attitudes for which there is no commonly agreed upon acceptable standard. 


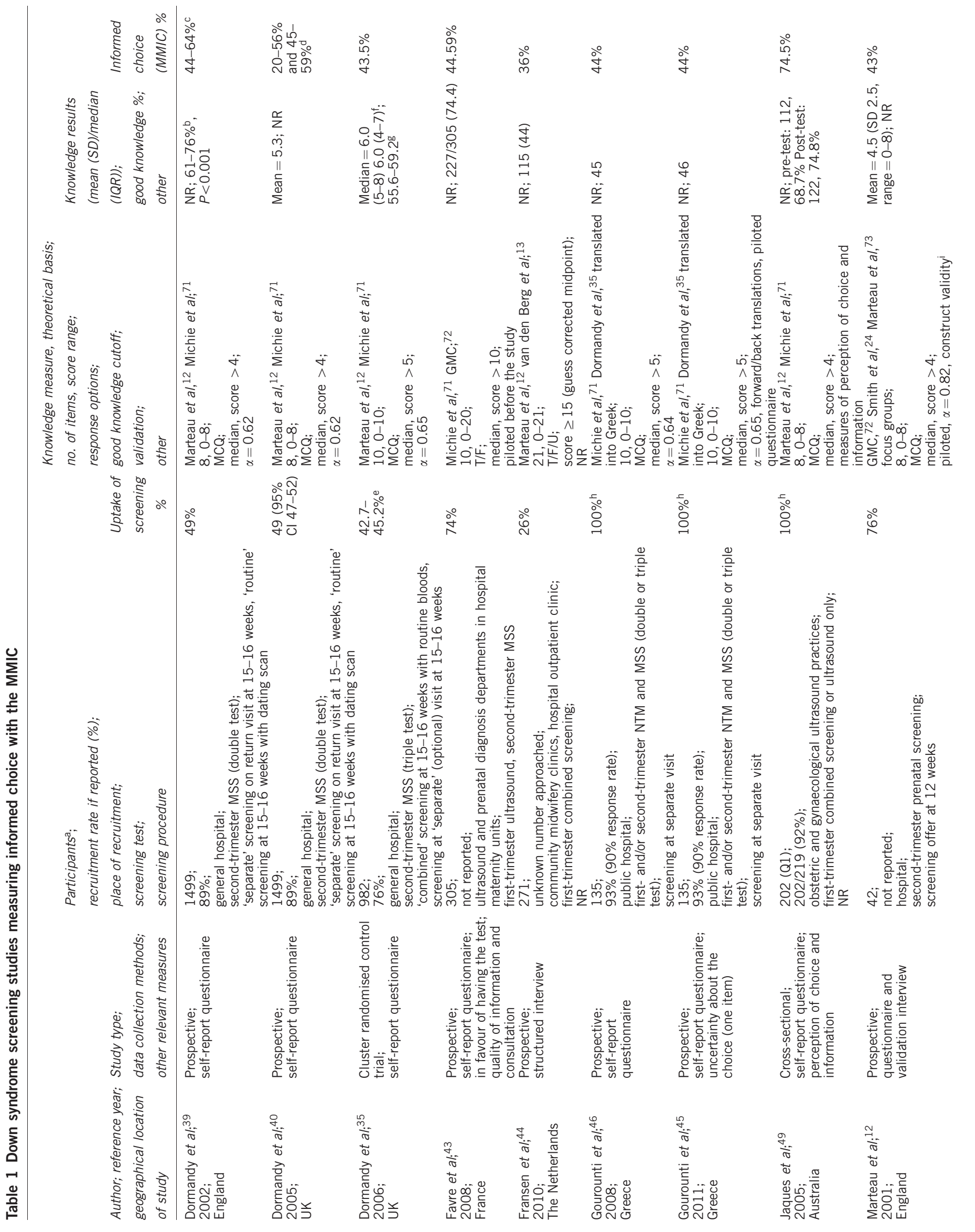




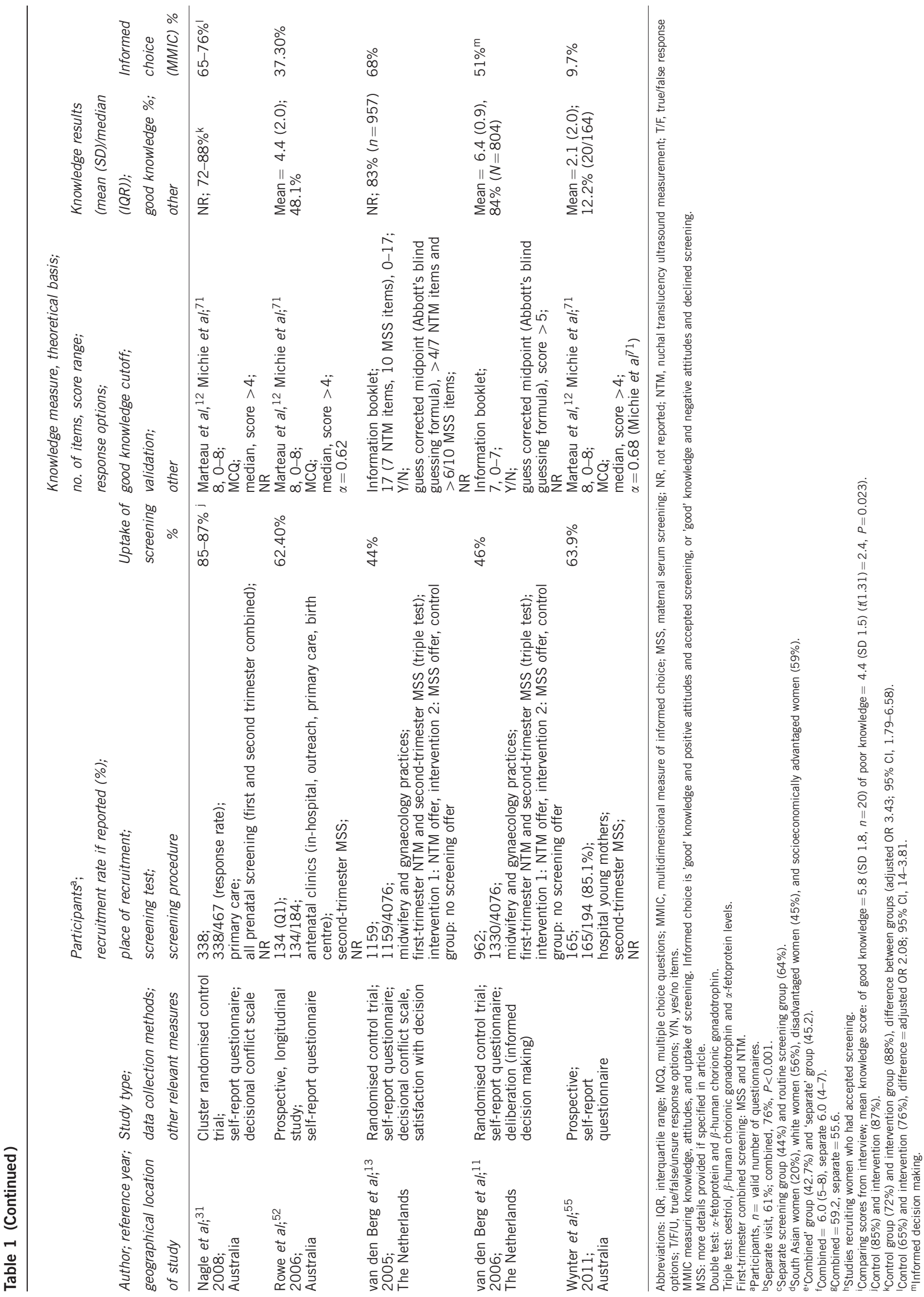



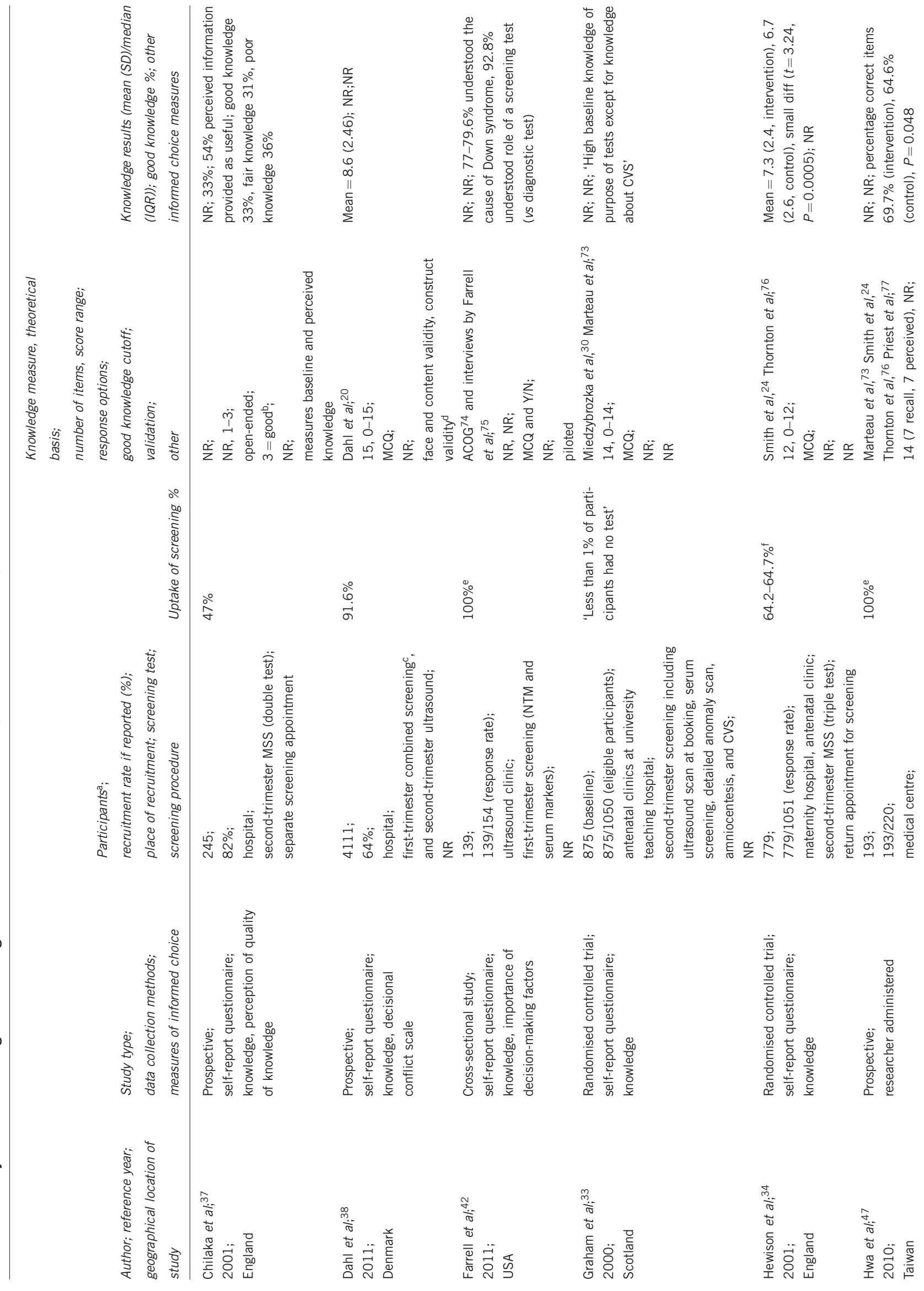


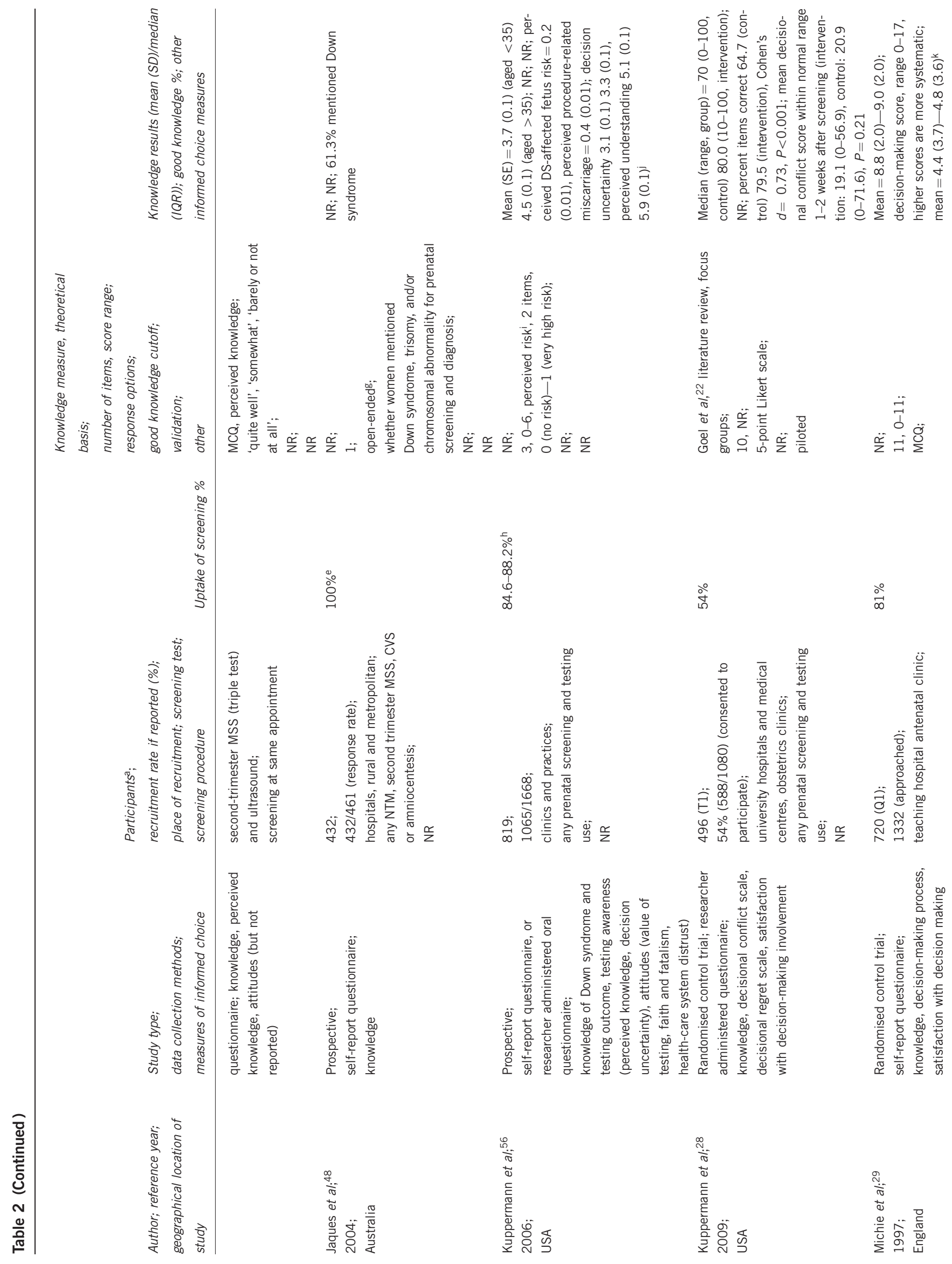




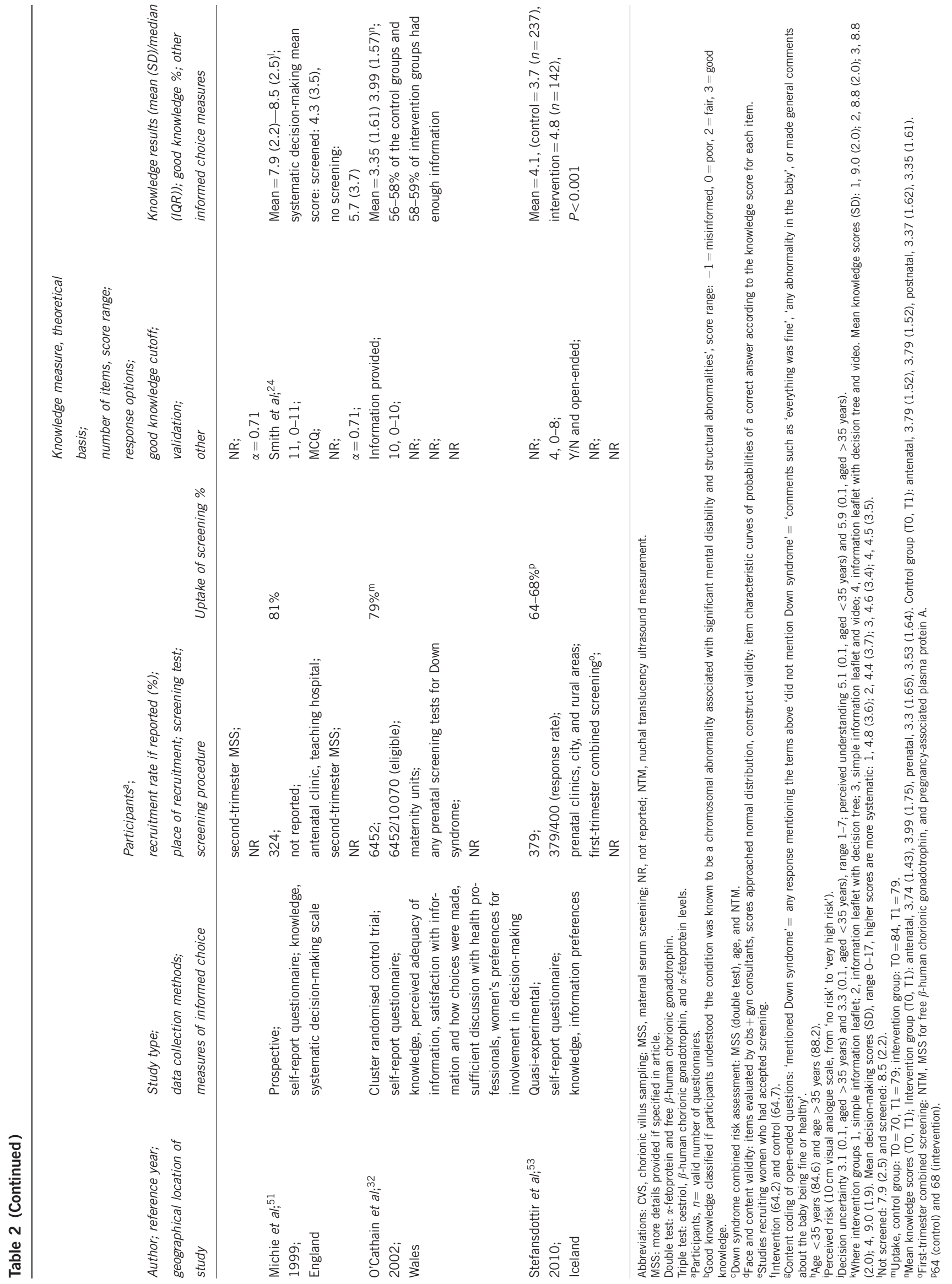




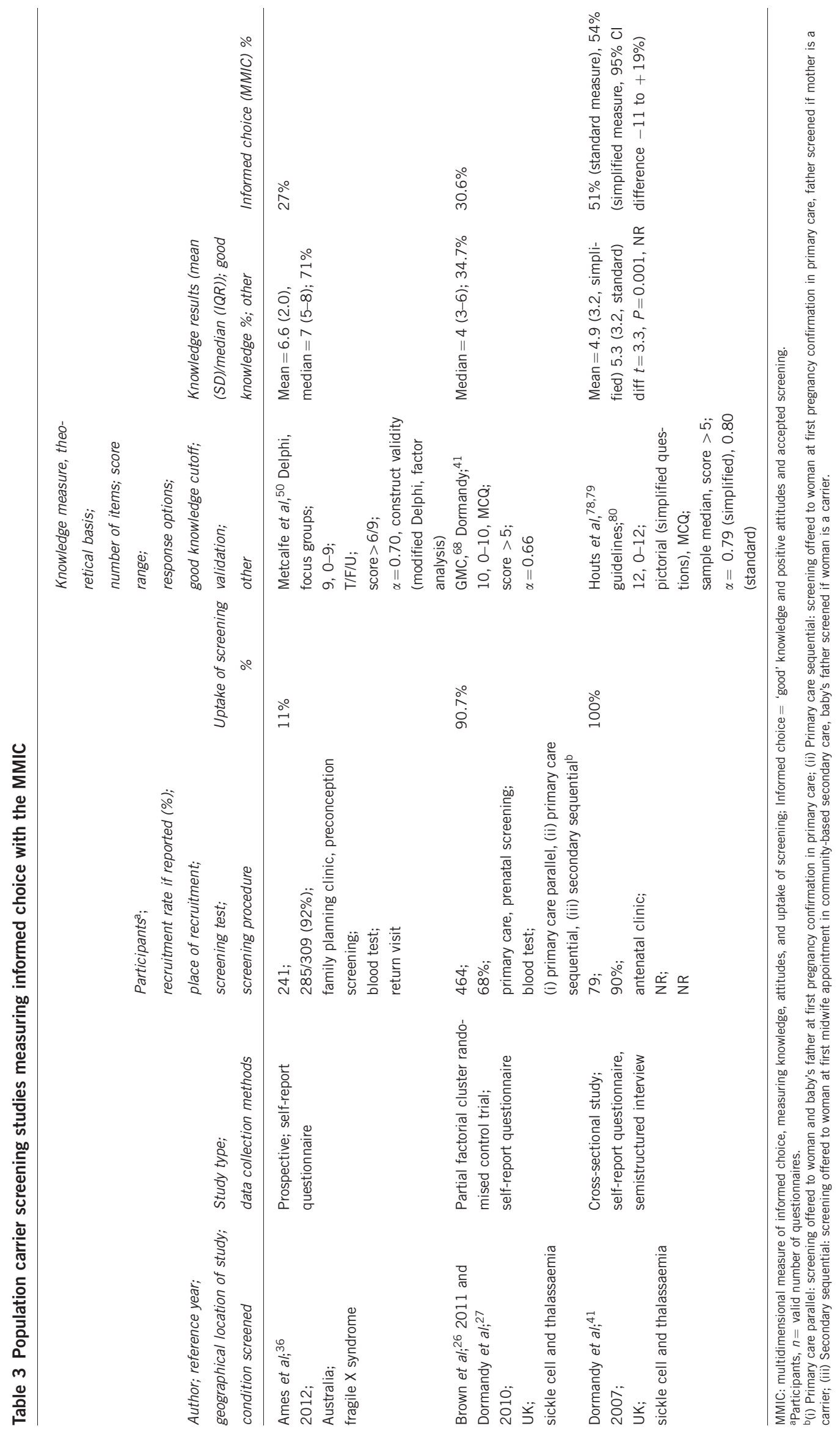




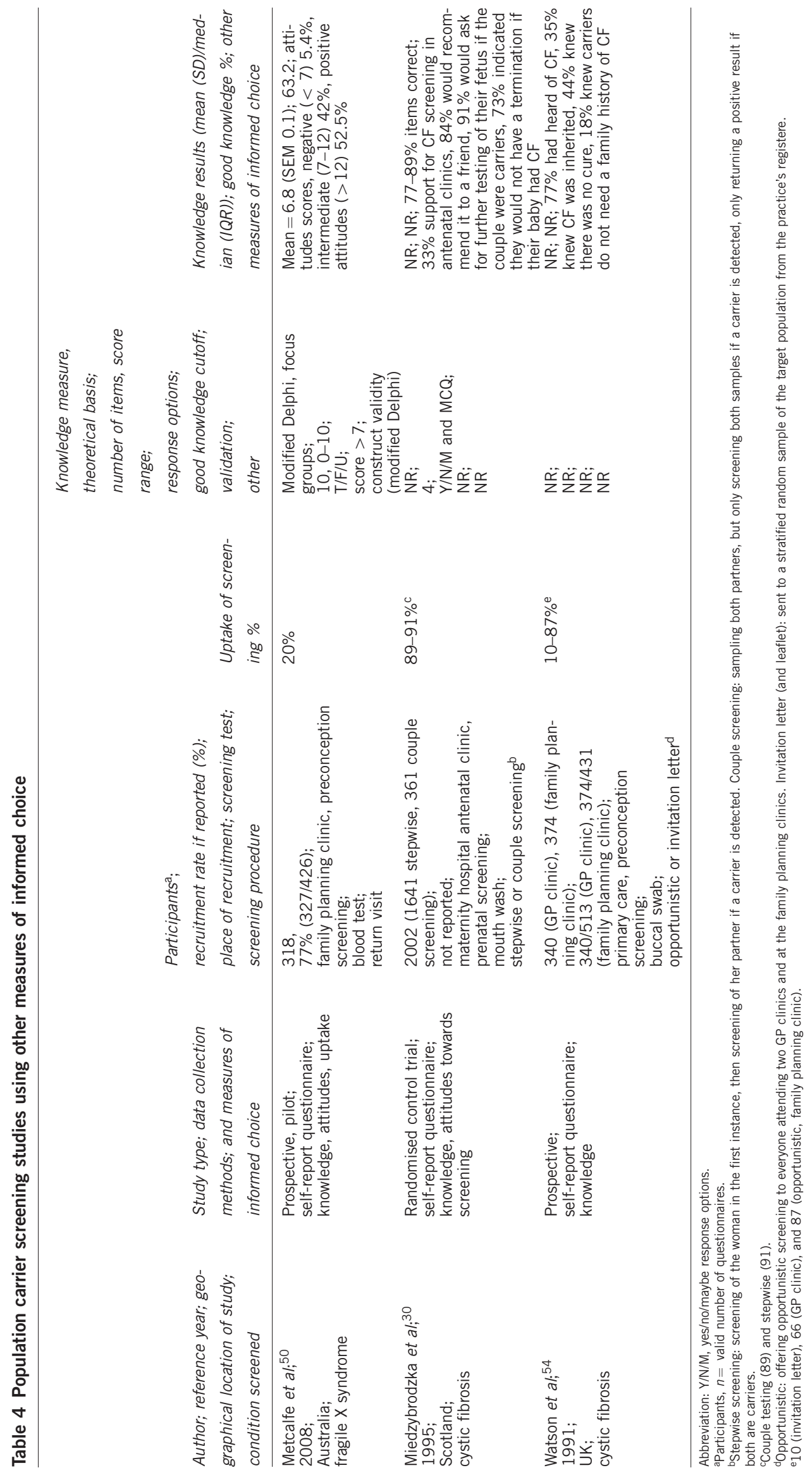


Good knowledge was defined in various ways using the median ${ }^{12}$ and a guess-corrected midpoint. ${ }^{13}$ Validation of the 'good' knowledge cutoff was generally lacking, with only two studies validating the cutoff using interview data. ${ }^{12,41}$

Informed choice varied for DS screening from $9.7 \%{ }^{55}$ to $74.5 \%{ }^{49}$ In prenatal $\mathrm{Hb}$ carrier screening, $30.4-50 \%$ were classified as having made an informed choice. ${ }^{26,41}$ Low rates of informed choice were generally because of poor knowledge. In preconception carrier screening for FXS, only $27 \%$ of participants were classified as having made an informed choice. ${ }^{36}$ In this setting, uninformed choices were due to low value consistency, largely because screening required people to return for a separate appointment to have blood taken, resulting in women having positive attitudes towards screening but not attending the return appointment to provide the blood sample for screening. ${ }^{50}$

\section{Perception of choice}

Five studies incorporated measures of participants' perception of their choice about the screening test. ${ }^{32,43,49,51,56}$ When screening is offered as part of 'routine' care, it can be interpreted by participants as being compulsory. However, ideally, screening should be offered in a way that promotes autonomy; being free from coercion. Assessing the subjective perception of choice is one way of assessing the degree of autonomy that individuals exercised when making their choice.

The items measuring perception of choice varied across studies; four studies used a single item, $, 32,43,49,56$ and one study included a single item within a systematic decision-making scale. ${ }^{51}$ All items asked women, in different ways, whether they thought the screening was optional, or who should make their choice about screening.

In prenatal screening for DS, almost half $(46 \%)$ of women thought screening was routine, ${ }^{48}$ and most $(82 \%)$ thought it was compulsory. ${ }^{43}$ However, most women reported believing that the choice should lie with the individual offered screening. ${ }^{56}$ Perceptions of choice are likely to be context specific; for example, in countries where screening is funded by the government or is socially accepted, testing might be seen as part of 'routine' care. ${ }^{38,46}$

\section{Attitudes and feelings towards screening}

Of the 33 studies, 5 used an attitudes scale different from that used in the MMIC, ${ }^{30,43,47,56}$ or the same scale but used independently of the MMIC. ${ }^{50}$ These studies used a variety of different items within the attitudes measures; three studies used various items regarding participants' general feelings towards screening, ${ }^{30,50}$ and whether or not the participant was in favour of DS screening. ${ }^{43}$ One study measured a broad range of attitudes and values towards screening such as 'faith or fatalism. ${ }^{28}$ Two studies also measured participants' acceptance and values relating to screening, termination of pregnancy, and having a child with the condition being screened. ${ }^{30,56}$ One study did not report the items or results of the attitudes measure. ${ }^{47}$

Attitudes regarding screening are particularly important to measure as it has been argued that screening choices ought to be aligned with the morals and values of the individual. ${ }^{3}$ These measures however need to be more consistent and validated within the sample population.

\section{Decisional conflict}

The decisional conflict scale (DCS) was used to evaluate participants' feelings regarding the choice outcome in five studies; four $\mathrm{RCTs}^{13,28,31,38}$ and an adapted version was used in one prospective study. ${ }^{56}$ The DCS was designed as a general scale, not for a particular condition, and validated with health-care consumers deciding about hypothetical scenarios of influenza vaccination and breast cancer screening. ${ }^{10}$ The scale comprises five subscales (information, values clarity, support, uncertainty, and effective decision) measuring the outcome of how an individual feels about a health-related decision, rather than evaluating whether the decision was an informed one. Decisional conflict is defined as 'a state of uncertainty about the course of action to take' that can occur when decisions are difficult, resulting in decisions that are not effective, and where an individual may feel uncertain about their decision. Rather, an effective decision is defined as one that is informed, consistent with personal values and acted upon. ${ }^{10}$ Decisional conflict is important in informed choice evaluation because it directly measures participants' perspectives of how certain they feel about the decision and their perceived autonomy in making the decision.

Results can be compared across the studies as the DCS is a standardised measure on a scale of 0-100 with validated cutoff scores for low decisional conflict (scores <25) and increased decisional conflict (scores > 37.5) ${ }^{10}$ Decisional conflict was reported within the normal range in two studies (mean ranging from 1.65 to 23.9 ) 28,31 with another study also demonstrating a relationship between low decisional conflict and high levels of knowledge (OR $=1.31,95 \% \mathrm{CI}$, $1.26-1.37, P<0.001){ }^{38}$

\section{Satisfaction with decision and decisional regret}

Five studies measured satisfaction with decision or decisional regret. ${ }^{13,28,29,32,50}$ Satisfaction and decision regret scales are explicit measures of how people feel about their choices, ${ }^{4}$ and can be used to measure the outcome of decision making. ${ }^{4,58}$ An informed decision may lead to better outcomes ${ }^{59}$ such as increased satisfaction, or low regret with the decision. ${ }^{4,10,60}$ Decision regret can be defined as 'remorse or distress over a decision' that can stem from 'knowledge that the choice made was nonoptimal'. ${ }^{4}$ Decision satisfaction and regret, however, are not the same as making an informed choice; someone may have low satisfaction but have made an informed choice. $^{59}$

Two studies used scales of decision satisfaction, ${ }^{13,29}$ one study used the decision regret scale, ${ }^{28}$ whereas two others used individual items to measure satisfaction with how the choices were made. ${ }^{32,50}$ In studies where satisfaction with screening choices was measured, most women were very satisfied with their choices, ${ }^{29}$ and did not regret their decisions. ${ }^{28,50}$ One study showed significantly higher satisfaction scores in people making informed decisions compared with uninformed decisions $(t=4.9, P<0.001, t=2.1, P<0.05) .{ }^{13}$

\section{Measures of systematic decision making}

Three studies used measures of systematic, informed decision making. ${ }^{11,29,51}$ According to a rational decision-making perspective, an informed decision needs to involve a systematic process of weighing up the advantages and disadvantages of the choice, that is, deliberating, as well as the decision being informed in order to have the best outcome. ${ }^{7}$ Informed decision making is differentiated from an informed choice, where the former involves the process of deliberation. Decisions that are not systematic are often made using mental short cuts or heuristics that can lead to biases in decision making and poorer quality decisions. ${ }^{61}$ To account for this aspect of decision making, an 18-item systematic decision-making scale was developed, ${ }^{29,51}$ and a 6-item deliberation scale was used with the existing MMIC. ${ }^{11}$ These measures have been well validated, ${ }^{51}$ indicating that each accurately measures the systematic decisionmaking construct. However, these scales have not been widely used to date. 


\section{DISCUSSION}

This systematic review evaluated how informed choice has been measured in population reproductive genetic screening. Studies offering population genetic screening should endeavour to measure informed choice as well as uptake of screening. ${ }^{2}$ The studies identified in this review demonstrate the array of methods used to measure informed choice in population reproductive genetic screening. Our findings highlight well-validated measures, and also some limitations and inconsistencies in evaluating informed choice in this setting.

The concept of informed choice is a multidimensional construct and has been defined in a numbers of ways. ${ }^{11,12,32,33,48}$ Measuring knowledge is integral for informed choice evaluation, yet there has been little development and validation of knowledge measures. A heterogeneous range of knowledge items were used in the studies reviewed. Even the reporting of validated knowledge scales varied within DS screening where there has been much development of informed choice measurement. ${ }^{12,31,39,40,46,49}$

There are no agreed thresholds or minimum standards for the knowledge an individual is required to have to make an 'informed' choice. As such, a variety of methods were used to classify participants as having 'good enough' knowledge for an informed choice. This is problematic because when good knowledge is defined differently, it is not possible to compare the proportion of informed choice across studies. Some measures, such as the MMIC, required the scales to be dichotomised. Dichotomising continuous scales into binary or categorical data has been criticised and is not recommended by some editorial guidelines. ${ }^{62}$

The authors of the MMIC drew on work by O'Brien-Pallas, integrating the definition of informed choice with the theoretical perspective of rational behaviour. ${ }^{57,63}$ The MMIC has been validated and applied widely in DS screening, ${ }^{11-13,31,35,39,40,43,44,49,52,55}$ and now has been used in $\mathrm{Hb}^{26,41}$ and FXS carrier screening. ${ }^{36}$ In nonpregnancy screening, the construct validity of the value-consistency domain needs to be validated because in nonpregnancy carrier screening individuals do not need to make a decision when offered screening, rather they may defer the decision to another time in their life. ${ }^{36,64}$ Therefore, the MMIC needs to be further explored and undergo validation in screening settings other than prenatal screening.

Another approach to encapsulate the informed choice construct is to use the DCS with a knowledge measure. The DCS is a well validated measure ${ }^{10}$ used in five studies measuring informed choice. ${ }^{13,28,31,38,56}$ Incorporating a knowledge scale as well, perhaps using 'good' knowledge, combined with a low decisional conflict could be one way of defining informed decision making. This way, participants' perspectives of whether they made an informed and free choice, and whether they were supported, are taken into consideration. These aspects are similar to other concepts such as the perception of choice and satisfaction, yet more research and agreement is needed to determine how these measures should be used and how they relate to evaluating informed choice.

When using the MMIC, or any measure out of its original context, the limitations of the measure need to be recognised until the measure has been validated within the new setting. The MMIC has been criticised for oversimplifying informed choice into a binary variable, potentially incorrectly classifying choices that are informed. ${ }^{52,65}$ The MMIC also does not take into account the cognitive processes involved in making a decision, such as deliberation. ${ }^{7,11}$ Although there are limitations to the MMIC, as for any measure, the MMIC remains the only single, multidimensional measure of informed choice for genetic screening. The MMIC is a relatively short instrument to administer and easy to use, with many published studies detailing how to apply and interpret the measure. However, the MMIC does require a knowledge measure that is specific to the condition being tested.

In the future, a knowledge measure may need to address the general phenotype (multiple congenital anomalies, developmental delay, reduced life expectancy, and so on), ${ }^{66}$ rather than be condition specific. With multiplex genetic technologies now enabling screening for multiple conditions, ${ }^{67}$ measuring informed choice with conditionspecific knowledge scales is problematic. With this technology becoming available directly to consumers, there is a need for more research about informed choice in the general population, and outside the prenatal context. There is also a need for ongoing evaluation and publication of informed choice in existing programmes, with implementation of changes where needed to facilitate informed choice. Moving forward, qualitative and mixed methods studies could be used to help understand informed choice in genetic screening and further inform how best to measure informed choice in the future.

Although we have applied rigorous systematic review methods, there are some limitations to this study. As informed choice is defined in a number of ways, it was difficult to develop a search strategy to locate all studies for this review. The search terms may not have included every definition and possible way that informed choice could be indexed in the databases. To account for this, we used citation tracking and contacted corresponding authors and experts in the field, and this yielded 13 additional relevant papers. The studies identified in the review came from a limited number of countries; almost half of the studies (14/33) were from the United Kingdom where informed choice is a key component of prenatal screening guidelines, ${ }^{68}$ and where the widely used measure, MMIC, was developed. Informed choice may be a component of guidelines and the evaluation of screening programmes in other countries, but the evaluations may not be published and hence not included in this review. Because of the heterogeneity of included studies, we were only able to conduct a narrative synthesis. However, our review has highlighted important challenges for this field of research and suggests the need for further work in the area especially with the expansion of carrier screening and noninvasive prenatal screening. ${ }^{69,70}$

The focus of this review was on the measurement tools used in population genetic screening for reproductive risk. For this reason the review did not include guidelines or qualitative studies. However, qualitative studies are important for exploring how individuals offered screening perceive informed choice. In fact, qualitative research could contribute to understanding issues of informed choice evaluation such as what is good knowledge, and whether individual's perceptions of informed choice reflect how informed choice is measured. This approach could be especially useful for new screening programmes that have not been evaluated.

This review has highlighted that there is no gold standard for evaluating good knowledge or informed choice in population reproductive genetic screening. Knowledge and autonomy are key aspects of informed choice; it is important to define what is important for participants to understand when accepting or declining screening and for individuals to understand that screening is optional and their own personal choice. A dearth of studies involving future fathers and partners suggests more research is needed to better understand their role, and women's perspectives about this in making choices about screening. There are a variety of informed choice 
measures with variable quality that would benefit from further development and evaluation. Results of informed choice evaluations must be presented in a meaningful way to indicate the programme's efficacy to facilitate informed choice. Particular attention ought to be directed towards addressing how 'good' knowledge and informed choice are defined and measured from the outset of a screening programme.

\section{CONFLICT OF INTEREST}

The authors declare no conflict of interest.

\section{ACKNOWLEDGEMENTS}

We thank Pho Chua, librarian at the Royal Children's Hospital, for her assistance. We acknowledge the funding enabling this research from the Murdoch Childrens Research Institute, Australian Postgraduate Award, and the Victorian Government's Operational Infrastructure Support Programme.

1 Khoury MJ, McCabe LL, McCabe ERB: Genomic medicine - Population screening in the age of genomic medicine. N Engl J Med 2003; 348: 50-58.

2 Godard B, ten Kate L, Evers-Kiebooms G, Ayme S: Population genetic screening programmes: principles, techniques, practices, and policies. Eur J Hum Genet 2003; 11: S49-S87.

3 World Health Organisation: Proposed International Guidelines on Ethical Issues in Medical Genetics and Genetic Services. Geneva, 1998.

4 Brehaut JC, O'Connor AM, Wood TJ et al: Validation of a decision regret scale. Med Decis Making 2003; 23: 281-292.

5 Wertz D, Fletcher J, Berg K, Boulyjenkov V: Guidelines on ethical issues in medical genetics and the provision of genetics services, Hereditary Diseases Program, Division of Noncommunicable DiseasesGeneva: World Health Organisation, 1995.

6 Kohut RJ, Dewey D, Love EJ: Women's knowledge of prenatal ultrasound and informed choice. J Genet Counsel 2002; 11: 265-276.

7 Bekker H, Thornton JG. Airey CM et al: Informed decision making: an annotated bibliography and systematic review. Health Technol Assess 1999; 3: 1-156.

8 Briss P, Rimer B, Reilley B et al: Promoting informed decisions about cancer screening in communities and healthcare systems. Am J Prev Med 2004; 26: 67-80.

9 Rimer BK, Briss PA, Zeller PK, Chan ECY, Woolf SH: Informed decision making: what is its role in cancer screening? Cancer 2004; 101: 1214-1228.

10 O'Connor AM: Validation of a decisional conflict scale. Med Decis Making 1995; 15: 25-30.

11 van den Berg M, Timmermans DRM, ten Kate LP, van Vugt JMG, van der Wal G: Informed decision making in the context of prenatal screening. Patient Educ Couns 2006; 63: 110-117.

12 Marteau TM, Dormandy E, Michie S: A measure of informed choice. Health Expect 2001; 4: 99-108.

13 van den Berg M, Timmermans DRM, Ten Kate LP, van Vugt JMG, van der Wal G: Are pregnant women making informed choices about prenatal screening? Genet Med 2005; 7: 332-338

14 Jepson RG, Hewison J, Thompson AGH, Weller D: How should we measure informed choice? The case of cancer screening. J Med Ethics 2005; 31: 192-196.

15 Irwig L, McCaffery K, Salkeld G, Bossuyt P: Informed choice for screening: implications for evaluation. Br Med J 2006; 332: 1148-1150.

16 Mullen PD, Allen JD, Glanz $\mathrm{K}$ et al: Measures used in studies of informed decision making about cancer screening: a systematic review. Ann Behav Med 2006; 32 188-201.

17 Foundation for Genetics and Population Health. Glossary of genetics terminology. 2012 (cited 08/09/2013); URL. http://www.phgfoundation.org/pages/resources/ glossary.htm

18 Rostant K, Steed L, O'Leary P: Survey of the knowledge, attitudes and experiences of Western Australian women in relation to prenatal screening and diagnostic procedures. Aust N Z J Obstet Gynaecol 2003; 43: 134-138.

19 De Vigan C, Vodovar V, Goujard J, Garel M, Vayssière C, Goffinet F: Mothers' knowledge of screening for trisomy 21 in 1999: a survey in Paris maternity units. Eur J Obstet Gynecol Reprod Biol 2002; 104: 14-20.

20 Dahl K, Hvidman L, Jørgensen FS et al: First-trimester Down syndrome screening: pregnant women's knowledge. Ultrasound Obstet Gynecol 2011; 38: 145-151.

21 Glazier R, Goel V, Holzapfel S, Summers A, Pugh P, Yeung M: Written patient information about triple-marker screening: a randomized, controlled trial. Obste Gynecol 1997; 90: 769-774.

22 Goel V, Glazier R, Holzapfel S, Pugh P, Summers A: Evaluating patient's knowledge of maternal serum screening. Prenat Diagn 1996; 16: 425-430.

23 Kaiser AS, Ferris LE, Pastuszak AL et al: The effects of prenatal group genetic counselling on knowledge, anxiety and decisional conflict: issues for nuchal translucency screening. J Obstet Gynaecol 2002; 22: 246-255.

24 Smith DK, Shaw RW, Marteau TM: Informed consent to undergo serum screening for Down's syndrome: the gap between policy and practice. Br Med J 1994; 309: 776
25 Tschudin S, Huang D, Mor-Gultekin H, Alder J, Bitzer J, Tercanli S: Prenata counseling-implications of the cultural background of pregnant women on information processing, emotional response and acceptance. Eur J Ultrasound 2011; 32:(Suppl 2) E100-E107.

26 Brown K, Dormandy E, Reid E, Gulliford M, Marteau T: Impact on informed choice of offering antenatal sickle cell and thalassaemia screening in primary care: a randomized trial. J Med Screen 2011; 18: 65-75.

27 Dormandy E, Bryan S, Gulliford MC et al: Antenatal screening for haemoglobinopathies in primary care: a cohort study and cluster randomised trial to inform a simulation model. The Screening for Haemoglobinopathies in First Trimester (SHIFT) trial. Health Technol Assess 2010; 14: 1-160.

28 Kuppermann M, Norton ME, Gates E et al: Computerized prenatal genetic testing decision-assisting tool: a randomized controlled trial. Obstet Gynecol 2009, 113: 53-63.

29 Michie S, Smith D, McClennan A, Marteau TM: Patient decision making: an evaluation of two different methods of presenting information about a screening test. $\mathrm{Br} \mathrm{J}$ Health Psychol 1997; 2: 317-326.

30 Miedzybrodzka ZH, Hall MH, Mollison J et al: Antenatal screening for carriers of cystic fibrosis: randomised trial of stepwise v couple screening. Br Med J 1995; 310 353-357.

31 Nagle C, Gunn J, Bell R et al: Use of a decision aid for prenatal testing of fetal abnormalities to improve women's informed decision making: a cluster randomised controlled trial. Br J Obstet Gynaecol 2008; 115: 339-347.

32 O'Cathain A, Walters SJ, Nicholl JP, Thomas KJ, Kirkham M: Use of evidence based leaflets to promote informed choice in maternity care: randomised controlled trial in everyday practice. Br Med J 2002; 324: 643-646.

33 Graham W, Smith P, Kamal A, Fitzmaurice A, Smith N, Hamilton N: Randomised controlled trial comparing effectiveness of touch screen system with leaflet for providing women with information on prenatal tests. Br Med J 2000; 320: 155-160.

34 Hewison J, Cuckle $\mathrm{H}$, Baillie $\mathrm{C}$ et al: Use of videotapes for viewing at home to inform choice in Down syndrome screening: a randomised controlled trial. Prenat Diagn 2001; 21 : 146-149.

35 Dormandy E, Michie S, Hooper R, Marteau TM: Informed choice in antenatal Down syndrome screening: a cluster-randomised trial of combined versus separate visit testing. Patient Educ Couns 2006; 61: 56-64.

36 Ames AG, Jaques A, Ukoumunne OC et al: Development of a fragile X syndrome (FXS) knowledge scale: towards a modified multidimensional measure of informed choice for FXS population carrier screening. Health Expect 2012; e-pub ahead of print 15 October 2012; doi:10.1111/hex.12009.

37 Chilaka VN, Konje JC, Stewart CR, Narayan H, Taylor DJ: Knowledge of Down syndrome in pregnant women from different ethnic groups. Prenat Diagn 2001; 21: 159-164.

38 Dahl K, Hvidman L, Jorgensen FS, Kesmodel US: Knowledge of prenatal screening and psychological management of test decisions. Ultrasound Obstet Gynecol 2011; 38 152-157.

39 Dormandy E, Hooper R, Michie S, Marteau T: Informed choice to undergo prenatal screening: a comparison of two hospitals conducting testing either as part of a routine visit or requiring a separate visit. J Med Screen 2002; 9: 109-114.

40 Dormandy E, Michie S, Hooper R, Marteau TM: Low uptake of prenatal screening for Down syndrome in minority ethnic groups and socially deprived groups: a reflection of women's attitudes or a failure to facilitate informed choices? Int J Epidemiol 2005; 34: 346-352.

41 Dormandy E, Tsui EYL, Marteau TM: Development of a measure of informed choice suitable for use in low literacy populations. Patient Educ Couns 2007: 66: 278-295.

42 Farrell RM, Nutter B, Agatisa PK: Meeting patients' education and decision-making needs for first trimester prenatal aneuploidy screening. Prenat Diagn 2011; 31 1222-1228.

43 Favre R, Moutel G, Duchange N et al: What about informed consent in first-trimester ultrasound screening for Down syndrome? Fetal Diagn Ther 2008; 23: 173-184.

44 Fransen MP, Essink-Bot ML, Vogel I, Mackenbach JP, Steegers EAP, Wildschut HIJ: Ethnic differences in informed decision-making about prenatal screening for Down's syndrome. J Epidemiol Community Health 2010; 64: 262-268.

45 Gourounti K, Sandal J: The validation and translation of Multidimensional Measure of Informed Choice in Greek. Midwifery 2011; 27: 170-173.

46 Gourounti K, Sandall J: Do pregnant women in Greece make informed choices about antenatal screening for Down's syndrome? A questionnaire survey. Midwifery 2008, 24: 153-162.

47 Hwa HL, Huang LH, Hsieh FJ, Chow SN: Informed consent for antenatal serum screening for Down syndrome. Taiwan J Obstet Gynecol 2010; 49: 50-56.

48 Jaques AM, Halliday JL, Bell RJ: Do women know that prenatal testing detects fetuses with Down syndrome? J Obstet Gynaecol 2004; 24: 647-651.

49 Jaques AM, Sheffield LJ, Halliday JL: Informed choice in women attending private clinics to undergo first-trimester screening for Down syndrome. Prenat Diagn 2005 25: 656-664.

50 Metcalfe S, Jacques A, Archibald A et al: A model for offering carrier screening for fragile $X$ syndrome to nonpregnant women: results from a pilot study. Genet Med 2008; 10: 525-535.

51 Michie S, Smith D, Marteau TM: Prenatal tests: how are women deciding? Prenat Diagn 1999; 19: 743-748.

52 Rowe HJ, Fisher JRW, Quinlivan JA: Are pregnant Australian women well informed about prenatal genetic screening? A systematic investigation using the Multidimensional Measure of Informed Choice. Aust N Z J Obstet Gynaecol 2006; 46: 433-439. 
53 Stefansdottir V, Skirton H, Jonasson K, Hardardottir H, Jonsson JJ: Effects of knowledge, education, and experience on acceptance of first trimester screening for chromosomal anomalies. Acta Obstet Gynecol Scand 2010; 89: 931-938.

54 Watson EK, Mayall E, Chapple J et al: Screening for carriers of cystic fibrosis through primary health care services. Br Med J 1991; 303: 504-507.

55 Wynter KH, Rowe HJ, Fisher JR, Lee M, Quinlivan JA: Are adolescents' decisions about prenatal screening for Down syndrome informed? A controlled, prospective study. J Pediatr Adolesc Gynecol 2011; 24: 29-34.

56 Kuppermann M, Learman LA, Gates E et al: Beyond race or ethnicity and socioeconomic status: predictors of prenatal testing for Down syndrome. Obstet Gynecol 2006; 107: 1087-1097.

57 Ajzen I: The theory of planned behavior. Organ Behav Hum Decis Process 1991; 50: 179-211.

58 Green JM, Hewison J, Bekker HL, Bryant LD, Cuckle HS: Psychosocial aspects of genetic screening of pregnant women and newborns: a systematic review. Health Technol Assess 2004; 8: 1-128.

59 Bekker HL, Hewison J, Thornton JG: Understanding why decision aids work: linking process with outcome. Patient Educ Couns 2003; 50: 323-329.

60 Holmes-Rovner M, Kroll J, Schmitt $N$ et al: Patient satisfaction with health care decisions: the satisfaction with decision scale. Med Decis Making 1996; 16: 58-64.

61 Tversky A, Kahneman D: Judgment under uncertainty: heuristics and biases. Science 1974; 185: 1124-1131.

62 Dawson NV, Weiss R: Dichotomizing continuous variables in statistical analysis. Med Decis Making 2012; 32: 225-226.

63 O'Connor AM, O'Brien-Pallas L: Decisional conflict (specify); in Mcfarland GK, Mcfarlane EA (eds) Nursing Diagnosis \& Intervention: Planning for Patient Care. St Louis, MO: Mosby Incorporated, 1993; pp 468-478.

64 Archibald AD, Jaques AM, Wake S, Collins VR, Cohen J, Metcalfe SA: "It's something I need to consider": decisions about carrier screening for fragile $X$ syndrome in a population of non-pregnant women. Am J Med Genet A 2009; 149A: 2731-2738.

65 Kasparian NA, Wakefield CE, Meiser B: Assessment of psychosocial outcomes in genetic counseling research: an overview of available measurement scales. J Genet Counsel 2007; 16: 693-712.

66 Bunnik EM, Janssens ACJ, Schermer MH: A tiered-layered-staged model for informed consent in personal genome testing. Eur J Hum Genet 2012; 21: 596-601.
67 Counsyl. Genetic testing as the first step to a healthy pregnancy. 2013 (cited 21 November 2013); URL: www.counsyl.com

68 General Medical Council: Consent: patients and doctors making decisions together. General Medical Council: London, 2008.

69 Deans Z, Newson A: Should non-invasiveness change informed consent procedures for prenatal diagnosis? Health Care Anal 2011; 19: 122-132.

70 van den Heuvel A, Chitty L, Dormandy E et al: Will the introduction of non-invasive prenatal diagnostic testing erode informed choices? An experimental study of health care professionals. Patient Educ Couns 2010; 78: 24-28.

71 Michie S, Dormandy E, Marteau TM: The multi-dimensional measure of informed choice: a validation study. Patient Educ Couns 2002; 48: 87-91.

72 General Medical Council: Consent: patients and doctors making decisions together. General Medical Council: London, 1998.

73 Marteau TM, Johnston M, Plenicar M, Shaw RW, Slack J: Development of a selfadministered questionnaire to measure women's knowledge of prenatal screening and diagnostic tests. J Psychosom Res 1988; 32: 403-408.

74 American College of Obstetricians and Gynecologists Committee. ACOG Practice Bulletin No. 77: screening for fetal chromosomal abnormalities. Obstet Gynecol 2007; 109: 217-227.

75 Farrell RM, Dolgin N, Flocke SA, Winbush V, Mercer MB, Simon C: Risk and uncertainty: shifting decision making for aneuploidy screening to the first trimester of pregnancy. Genet Med 2011; 13: 429-436.

76 Thornton JG, Hewison J, Lilford RJ, Vail A: A randomised trial of three methods of giving information about prenatal testing. Br Med J 1995; 311: 1127-1130.

77 Priest JH, FitzGerald JM, Haag MM, Streets K, Vanisko M, Johnson JP: Acceptance of amniocentesis by women in the state of Montana (USA) who are screen positive for Down's syndrome. J Med Screen 1998; 5: 178-182.

78 Houts PS, Bachrach R, Witmer JT, Tringali CA, Bucher JA, Localio RA: Using pictographs to enhance recall of spoken medical instructions. Patient Educ Couns 1998; 35: 83-88.

79 Houts PS, Witmer JT, Egeth HE, Loscalzo MJ, Zabora JR: Using pictographs to enhance recall of spoken medical instructions II. Patient Educ Couns 2001; 43: 231-242.

80 US National Cancer Institute. Simplification of Informed Consent Documents. 2006. URL: http://www.nci.nih.gov/clinicaltrials/understanding/simplification-of-informedconsent-docs.

Supplementary Information accompanies this paper on European Journal of Human Genetics website (http://www.nature.com/ejhg) 